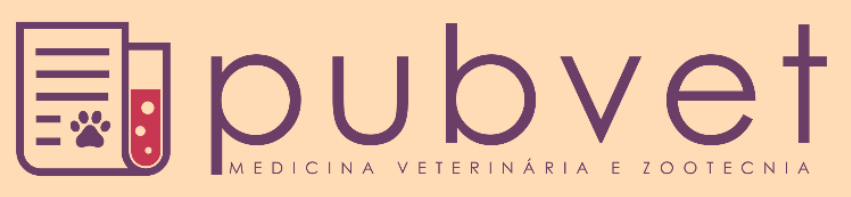

https://doi.org/10.31533/pubvet.v15n04a789.1-13

\title{
Estudo do uso de plantas medicinais na medicina veterinária em plataformas virtuais
}

\author{
Gabrielle Moura Nascimento ${ }^{1 *}{ }^{\ominus}$, Camila de Freitas Maia ${ }^{1} \bullet$, Bruno Silva Milagres ${ }^{2} \bullet$, Carlos \\ Alberto da Cruz Junior ${ }^{30}$
}

${ }^{I}$ Discente do curso de Medicina Veterinária, Centro Universitário de Brasília. Brasília-DF, Brasil.

${ }^{2}$ Professor do Centro Universitário de Brasília, Departamento de Medicina Veterinária. Brasília-DF, Brasil.

${ }^{3}$ Coodernador do curso de Medicina Veterinária do Centro Universitário de Brasília. Brasília-DF, Brasil.

*Autor para correspondência. E-mail: gabrielle.moura@sempreceub.com

\begin{abstract}
Resumo. Com o objetivo de verificar o uso de plantas medicinais nos animais, em grupos especializados na temática presentes no Facebook, WhatsApp e aplicativo DogHero, foi realizado um estudo transversal observacional descritivo entre os dias 17 e 23 de agosto de 2018 com o uso do Google Forms. Foram respondidos 124 questionários dos quais 53,22\% fizeram o uso de plantas medicinais como tratamento para animais. Todos os entrevistados que utilizaram as plantas receberam indicações, sendo 54,5\% de famílias e amigos, 31,5\% de médico veterinário, $9 \%$ por grupos em redes sociais e $5 \%$ buscaram por artigos. O maior número de respostas para o uso de plantas medicinais em animais foi do Distrito Federal com 56,92\% (região Centro-Oeste), seguindo de Pernambuco com 10,60\% (região Nordeste) e Rio Grande do Sul com 7,5\% (região Sul). Foram citadas utilizações em cães $(37 \%)$, roedores $(20 \%)$, felinos $(14 \%)$, répteis $(10 \%)$, aves $(9 \%)$, bovinos $(4 \%)$, equinos (4\%) e peixes (1\%). A camomila (Chamomilla recutita) foi a planta com maior relato de uso $(19,60 \%)$, seguida por $14,28 \%$ do chá-verde (Camellia sinensis), $10,78 \%$ da babosa (Aloe vera), 9,80\% do capim-santo (Cymbopogon citratus) e 9,80\% da erva-de-gato (Nepeta catari). O maior uso de plantas medicinais foi para o tratamento da ansiedade $(23,2 \%)$ seguido do tratamento de problemas no fígado e estômago e $10,4 \%$ para inchaço e $6,4 \%$ para obesidade. O cultivo próprio foi a principal forma de aquisição (36\%), seguida das farmácias de manipulação (28,78\%), feiras (18\%) e lojas (8\%). Conclui-se que o uso de plantas medicinais ocorre em diversas espécies de animais e nas mais distintas regiões do país, demonstrando a importância de se aprofundar os estudos e difusão da etnoveterinária.
\end{abstract}

Palavras-chave: fitoterápico, camomila, chá-verde

\section{Study of the use of medicinal plants in veterinary medicine on virtual platforms}

Abstract. In order to verify the use of medicinal plants in animals, in groups specialized in the theme present on Facebook, WhatsApp and DogHero application, a descriptive observational cross-sectional study was carried out between August 17 and 23, 2018 using Google Forms. 124 questionnaires were answered, of which 53.22\% used medicinal plants as a treatment for animals. All respondents who used the plants received indications, with $54.5 \%$ from families and friends, 31.5\% from veterinarians, $9 \%$ from groups on social networks and $5 \%$ from articles. The largest number of responses for the use of medicinal plants in animais was from the Federal District with $56.92 \%$ (Center-West region), followed by Pernambuco with $10.60 \%$ (Northeast region) and Rio Grande do Sul with $7.5 \%$ (South region). Uses were mentioned in dogs (37\%), rodents (20\%), felines (14\%), reptiles 
$(10 \%)$, birds $(9 \%)$, cattle (4\%), horses $(4 \%)$ and fish (1\%). Chamomile (Chamomilla recutita) was the plant with the highest reported use (19.60\%), followed by $14.28 \%$ of green tea (Camellia sinensis), $10.78 \%$ of aloe (Aloe vera), $9.80 \%$ of capim-santo (Cymbopogon citratus) and $9.80 \%$ of catnip (Nepeta catari). The greatest use of medicinal plants was for the treatment of anxiety (23.2\%) followed by the treatment of problems in the liver and stomach and $10.4 \%$ for bloating and $6.4 \%$ for obesity. Self-cultivation was the main form of acquisition (36\%), followed by handling pharmacies $(28.78 \%)$, fairs $(18 \%)$ and stores $(8 \%)$. It is concluded that the use of medicinal plants occurs in several species of animals and in the most distinct regions of the country, demonstrating the importance of deepening studies and disseminating ethnoveterinary.

Keywords: herbal medicine, chamomile, green tea

\section{Estudio del uso de plantas medicinales en medicina veterinaria en plataformas virtuales}

Resumen. Para verificar el uso de plantas medicinales en animales, en grupos especializados en la temática presente en Facebook, WhatsApp y la aplicación DogHero, se realizó un estudio observacional descriptivo transversal entre el 17 y 23 de agosto de 2018, mediante Google Forms. Se respondieron 124 cuestionarios, de los cuales el 53,22\% utilizaban plantas medicinales como tratamiento para animales. Todos los encuestados que utilizaron las plantas recibieron indicaciones, con 54,5\% de familiares y amigos, 31,5\% de médico veterinario, $9 \%$ de grupos en redes sociales y $5 \%$ de artículos. El mayor número de respuestas para el uso de plantas medicinales en animales fue del Distrito federal con 56,92\% (región Centro-Oeste), seguido de Pernambuco con 10,60\% (región Nordeste) y Rio Grande do Sul con 7,5\% (región sur). Se mencionaron usos en perros (37\%), roedores (20\%), felinos (14\%), reptiles (10\%), aves (9\%), bovinos (4\%), equinos (4\%) y peces $(1 \%)$. La manzanilla (Chamomilla recutita) fue la planta con mayor uso reportado $(19,60 \%)$, seguida del 14,28\% de té verde (Camellia sinensis), el 10,78\% de aloe (Aloe vera), 9,8\% de capim-santo (Cymbopogon citratus) y $9,80 \%$ de hierba gatera (Nepeta catari). El mayor uso de plantas medicinales fue para el tratamiento de la ansiedad $(23,2 \%)$, seguido del tratamiento de problemas en el hígado y estómago y el 10,4\% para la hinchazón y el 6,4\% para la obesidad. El autocultivo fue la principal forma de adquisición (36\%), seguido de las farmacias de manipulación $(28,78 \%)$, ferias $(18 \%)$ y tiendas $(8 \%)$. Se concluye que el uso de plantas medicinales se da en varias especies de animales y en las más distintas regiones del país, demostrando la importancia de profundizar en los estudios y difundir la etnoveterinaria.

Palabras clave: fitoterapia, manzanilla, té verde

\section{Introdução}

A biodiversidade é constituída por alguns elementos e, dentre eles, estão as plantas medicinais, que começaram a ser utilizadas na farmacologia como matéria-prima para a fabricação de fitoterápicos. Existem registro do uso de plantas na época do faraó Ramsés em 1500 a.C. sendo utilizadas na medicina e na astrologia. No Brasil, as primeiras referências foram dadas pelo padre José de Anchieta que formulou receitas à base de plantas para o tratamento de doenças (Lameira, 2008). Nos dias atuais, o uso de plantas vem crescendo rapidamente em tratamentos caseiros (chás, xaropes, tinturas, inalações e vinho medicinal) e na busca de novos métodos estruturais de princípios ativos naturais de interesse da indústria química e farmacêutica, além de serem bem acessíveis, muitas vezes sendo encontradas no quintal de casas, ervarias e lojas de produtos naturais. E com isso, os Órgãos Governamentais de Saúde Pública criaram meios de preservação para o uso de plantas ineficazes ou tóxicas, além das falsificações e adulterações, pois o uso incorreto de medicamentos fitoterápicos pode trazer efeitos colaterais (Lameira, 2008).

Para alguns autores, as plantas medicinais são todas aquelas que quando aplicada em um ser humano ou animal deve exercer uma ação terapêutica, auxiliando o organismo a normalizar funções fisiológicas 
prejudicadas, restaurar a imunidade enfraquecida, promover a desintoxicação e o rejuvenescimento. Este algo atuante é chamado de princípio ativo, seja ele composto de uma única substância existente na planta ou de um conjunto que agem sinergicamente, chamados de complexo fitoterápico (Firmo et al., 2012; Haraguchi \& Carvalho, 2010). Todavia, para a planta ser considerada verdadeiramente medicinal, é necessário que seja identificado o seu princípio ativo e que ele seja avaliado farmacologicamente (Lorenzi et al., 2002).

Com o avanço de uso de plantas medicinais na medicina humana, permitiu-se o uso na veterinária, sendo utilizada por muitos criadores, fazendeiros e veterinários na prevenção ou tratamento de enfermidades em animais domésticos. A fitoterapia na veterinária acaba sendo utilizada por ter algumas vantagens, pois acaba sendo menos invasiva, o medicamento pode ser ministrado de várias formas, baixo custo e alta disponibilidade. O uso desses conhecimentos populares na saúde animal é chamado de etnoveterinária (Batista et al., 2017), que estuda as práticas populares a favor da saúde e bem-estar animal e tem como tratamento produtos de origem animal, mineral e principalmente plantas medicinais (Monteiro et al., 2012; Teixeira et al., 2015).

Portanto, este estudo teve como principal objetivo avaliar o uso de plantas medicinais na medicina veterinária como tratamento contra doenças e infecções e quais as partes das plantas foram utilizadas.

\section{Material e métodos}

Foi realizado um estudo transversal observacional descritivo por meio de aplicação de questionário (Apêndice A) a fim de descrever sobre o uso de plantas medicinais na Medicina Veterinária. $\mathrm{O}$ questionário foi produzido no Formulários Google e aplicado em grupos de plantas medicinais presentes na rede social Facebook, grupos de WhatsApp e DogHero - aplicativo de celular de hospedagem para animais de companhia de todo Brasil, tendo iniciado no dia 17 de agosto e sendo finalizado no dia 23 de agosto de 2018.

As variáveis pesquisadas selecionadas no questionário foram: pessoas que já fizeram ou nunca fizeram uso de plantas para tratamento veterinário e senão, se tinham conhecimento sobre o assunto; espécie de animais; sexo; estados; plantas utilizadas (foram escolhidas 5 plantas de acordo com a porcentagem de sua utilização); parte da planta; doenças tratadas; tempo de uso; indicação; local de aquisição; se houve resultado e porquê utilizou esse tipo de tratamento.

Após a coleta de dados, os mesmos foram tabulados com auxílio do programa Microsoft Excel e onde foram elaborados os gráficos. Utilizou-se o programa Microsoft Word para cálculos em porcentagens.

\section{Resultados e discussão}

Para este estudo foram entrevistados 128 indivíduos em grupos de Plantas Medicinais no Facebook e no aplicativo DogHero; porém, houve um viés de três entrevistados que são estrangeiros e um que utilizou floral (que são a base de flores e são considerados tratamentos alternativos por não possuírem evidencia cientifica, enquanto medicamentos fitoterápicos e são considerados tratamentos complementares por possuírem conhecimento científico) e homeopáticos (são a base do reino vegetal, mineral e animal, não sendo $100 \%$ natural como os medicamentos fitoterápicos). Desta forma, os dados foram baseados somente em 124 entrevistados, no qual 53,2\% fizeram o uso de plantas como tratamento veterinário e dentre eles: $52,4 \%$ conheciam e $0,80 \%$ desconheciam. Entre os $46,8 \%$ dos entrevistados que não utilizaram plantas como tratamento, $24,2 \%$ já ouviram falar e $22,6 \%$ desconheciam o método (Gráfico 1). O conhecimento sobre plantas medicinais sempre esteve acompanhando a evolução do ser humano. Na qual civilizações primitivas descobriram o uso de plantas, não só como alimento, mas também para o tratamento contra doenças e o potencial de cura (Firmo et al., 2012). O conhecimento sobre tratamento com plantas é fundamental para famílias rurais por falta de recursos para cuidar da saúde. Pessoas que desconhecem, mas fazem o uso de plantas medicinais como tratamento, recorrem a esse método, na maioria das vezes, por não ter uma boa condição financeira para custear com os medicamentos para tratamentos nos animais (Scholl \& Ricardo, 2010). Segundo Yarza (1982), médicos desconhecem o uso de plantas medicinais e na maioria das vezes não fazem receitas com medicamentos fitoterápicos, assim não há conhecimento sobre o assunto. Enquanto algumas pessoas não utilizam, 
mesmo tendo o conhecimento, por não acreditarem que o tratamento fará efeito e que haverá chance de cura (Morais et al., 2005).

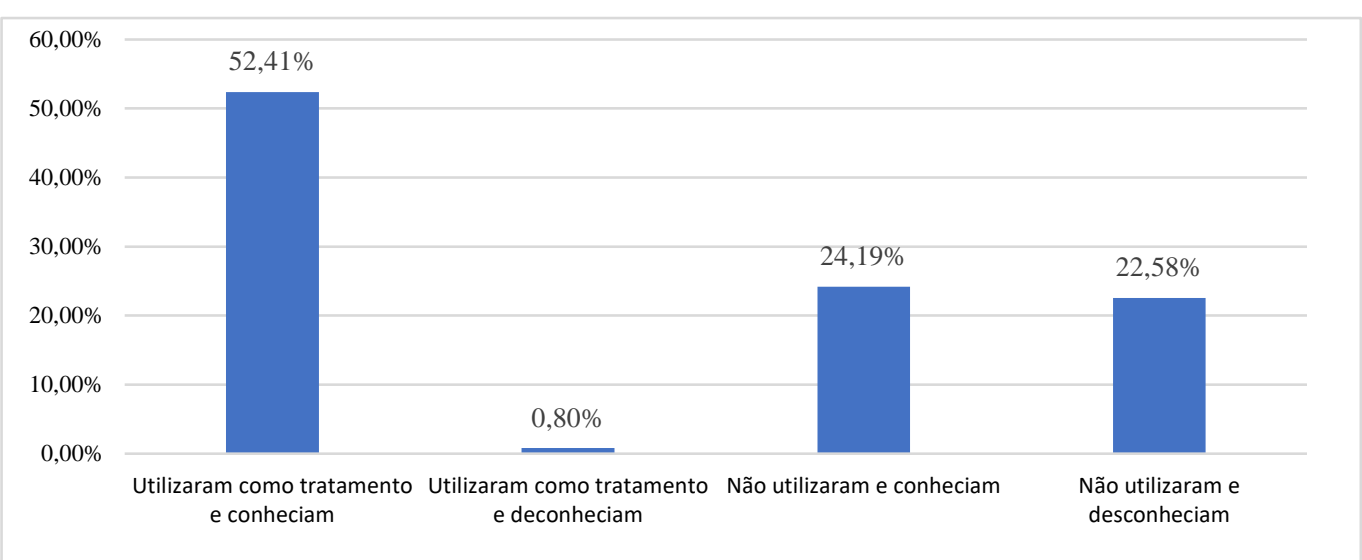

Gráfico 1. Porcentagem (\%) dos entrevistados que utilizaram plantas medicinais como tratamento e dos que não utilizaram, se conheciam sobre o método

Diante da questão de quais espécies de animais foi utilizado, o tratamento com plantas medicinais, dos 53,2\% dos entrevistados que fizeram tratamento com plantas medicinais apresentaram mais de uma espécie, sendo que um deles deixou de apresentar. Foram citados cães (37\%), roedores (20\%), felinos $(14 \%)$, répteis $(10 \%)$, aves $(9 \%)$, bovinos $(4 \%)$, equinos $(4 \%)$, baratas $(1 \%)$ e peixes $(1 \%)$ como mostrado no gráfico 2 .

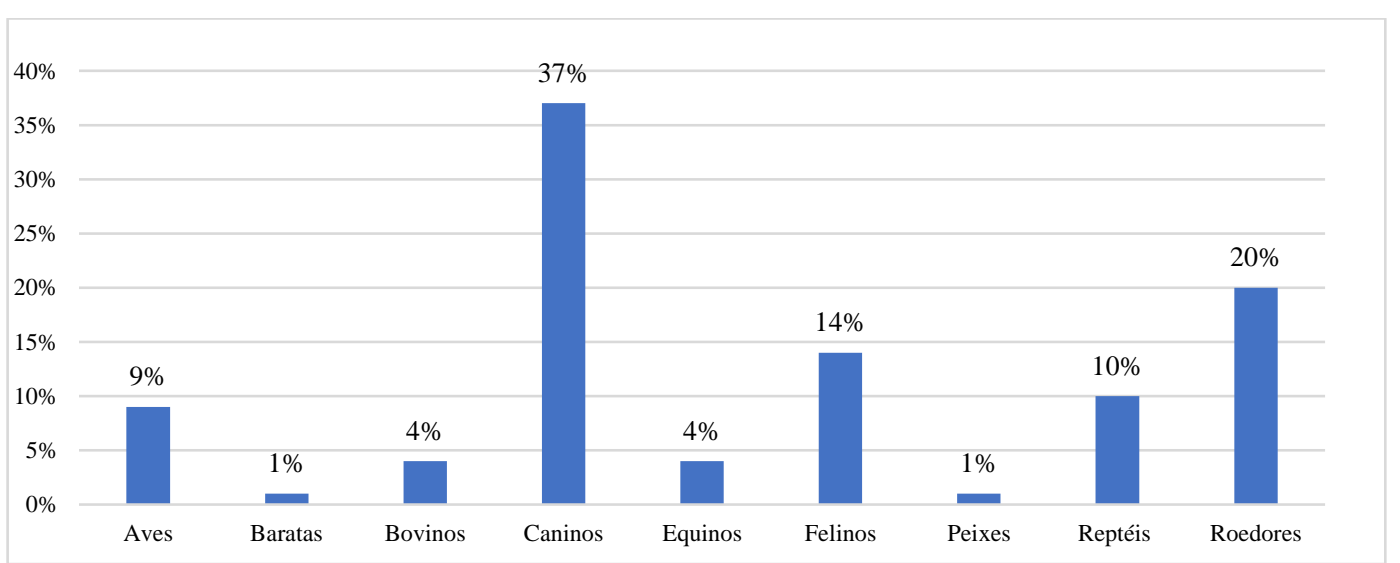

Gráfico 2. Espécies de animais que utilizaram plantas medicinais como tratamento (\%)

Segundo Simoni (2011), as plantas medicinais são usadas para tratar doenças em animais domésticos (companhia e produção), não sendo muito utilizadas para outras espécies. Todavia, alguns veterinários utilizam a fitoterapia como tratamento para animais silvestres (Sousa et al., 2020).

Das espécies apresentadas, 55,9\% eram fêmeas, $38,1 \%$ machos e 5,9\% eram indefinidos por serem aves. Houve um viés de 16 entrevistados que não informaram o sexo do animal (Figura 1). A sexagem das aves é importante, pois a maioria das aves existentes no mundo não possuem dimorfismo sexual e, quando possuem, é muito sutil, podendo ocorrer somente no período de maturidade sexual (Vieira et al., 2009). Não foram encontrados relatos de casos do uso de plantas medicinais como tratamento relacionados ao gênero sexual em animais. Entretanto em humanos, a procura do tratamento com plantas medicinais é mais procurada por mulheres por conta de indicações ginecológicas (Silva et al., 2016).

Os estados que mais apresentaram maiores índices de tratamento com plantas medicinais, apresentados no mapa (Figura 2), foram Distrito Federal com 56,9\% (região Centro-Oeste), Pernambuco com 10,6\% (região Nordeste) e Rio Grande do Sul com 7,5\% (região Sul). Atualmente, todas as regiões do Brasil vêm aumentando o conhecimento e o cultivo de plantas medicinais (Lorenzi et al., 2002). Todavia, as indústrias de medicamentos fitoterápicos, que utiliza como matéria-prima plantas, estão mais aglomeradas nas regiões Sul e Sudeste do Brasil (Carvalho et al., 2008), por isso ocorre um grande porcentual nos estados citados. Na região Norte do Brasil, os produtores rurais começaram um grande 
cultivo de plantas medicinais para uso próprio e como fonte de renda (Silva et al., 2013). Nos dias atuais, Brasília se tornou pioneira na produção de medicamentos fitoterápicos que chegam para a população por meio da Farmácia Viva do Ministério da Saúde e entre as regiões do entorno de Brasília que possuem uma assistência com a rede pública para o recebimento desses medicamentos está Sobradinho (Lourenzani et al., 2004). Algumas plantas Medicinais, por exemplo, a camomila (Chamomilla recutita) é amplamente cultivada no Sul e no Sudeste do Brasil. Enquanto a babosa (Aloe vera) cresce de uma forma gigantesca no Nordeste (Lorenzi et al., 2002).

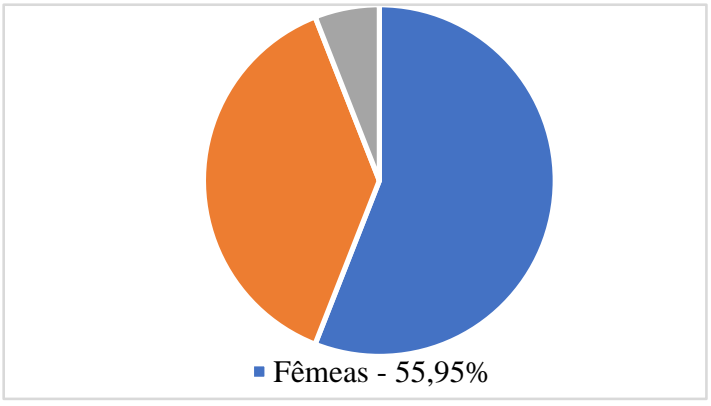

Figura 1. Sexo biológico das espécies dos animais que utilizaram plantas medicinais como tratamento.

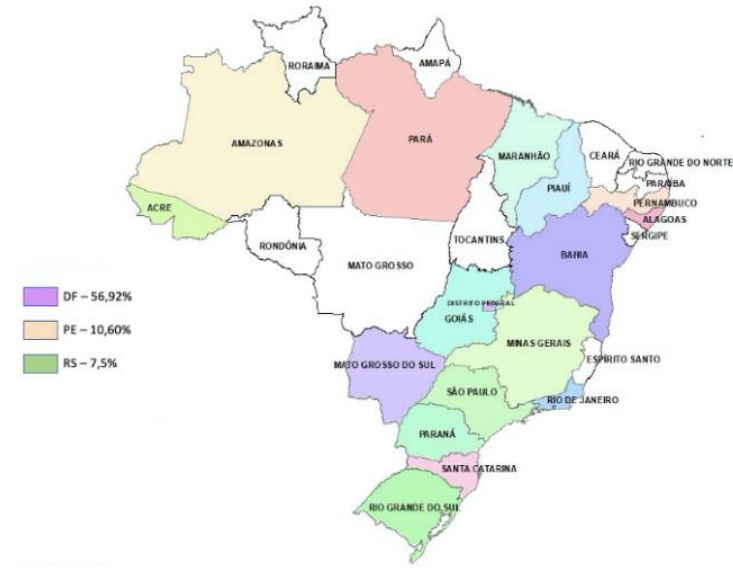

Figura 2. Mapa dos estados que mais apresentaram índices de tratamento com plantas medicinais (\%).

Nos entrevistados que fizeram o tratamento em seus animais, entre eles, houve um viés de um que se esqueceu de qual planta utilizou. Os entrevistados citaram mais de duas plantas como tratamento e cinco foram selecionadas por apresentarem um índice maior de uso, sendo: 19,6\% camomila (Chamomilla recutita), 14,3\% chá-verde (Camellia sinensis), 10,8\% babosa (Aloe vera), 9,8\% capimsanto (Cymbopogon citratus) e 9,8\% erva-de-gato (Nepeta cataria). Houve um viés de $1 \%$ que não se recorda de qual planta foi utilizada para o tratamento (Gráfico 3). A camomila é uma das plantas que é mais usada pela medicina tradicional europeia e está incluída nas Farmacopeias de quase todos os países. A infusão aquosa das flores e do óleo essencial são empregados em cremes e pomadas para promover a cicatrização da pele e por isso possui um índice alto de seu uso (Lorenzi et al., 2002), sendo que na maioria dos animais, é mais usada como antibactericida (Ozaki \& Duarte, 2006).

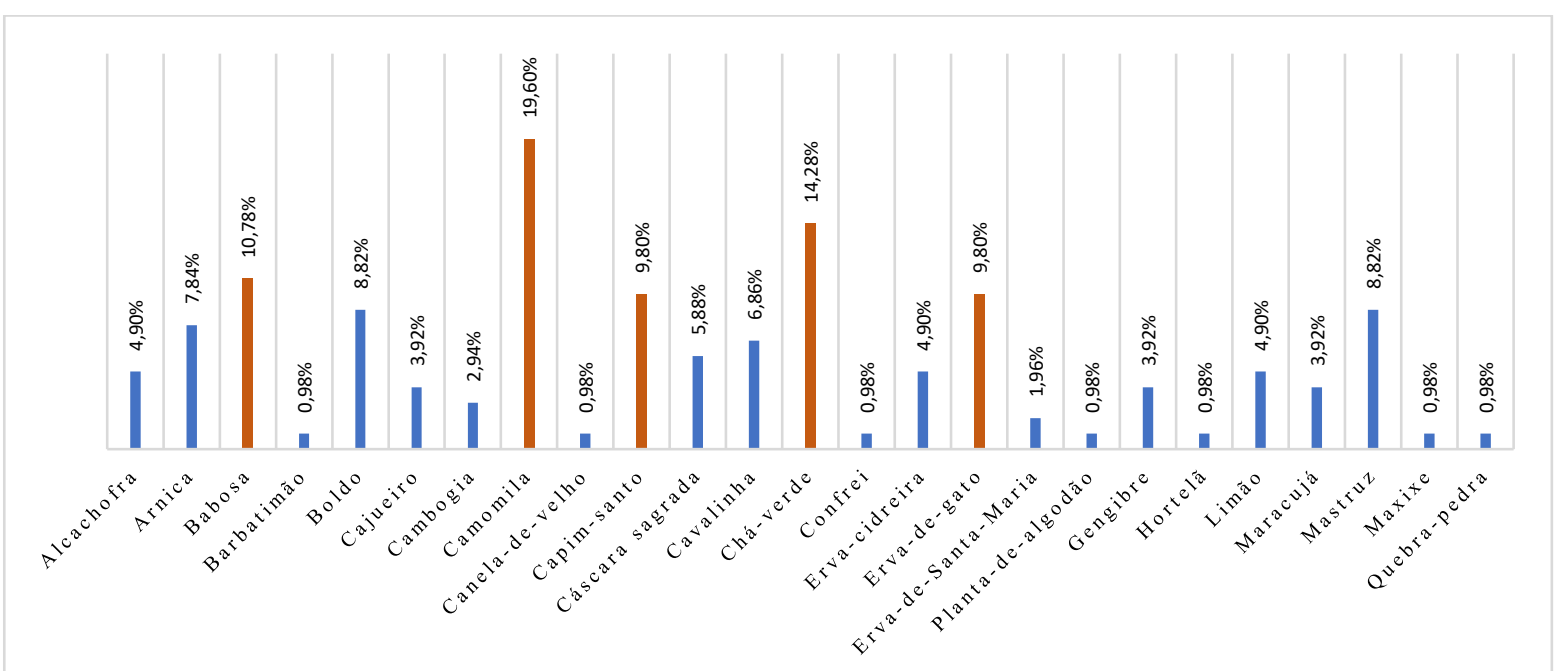

Gráfico 3. Porcentual (\%) das plantas mais utilizadas para o tratamento de doenças ou infecções em animais, sendo que cinco foram escolhidas por apresentarem um índice maior de uso

O chá-verde é produzido por meio de dissecação após sua fermentação (Pateiro et al., 2014). Em humanos, é utilizada como bebida semelhante parecida com o café. E segundo a literatura, o uso do chá 
reduz a incidência de câncer no aparelho gastrointestinal (Gonçalves et al., 2015). Nos animais, seus componentes possuem um grande significativo contra diabetes e estudos comprovam que os polifenóis do chá verde auxiliam na perda de peso, na qual já foi testado em ratos com obesidade. Os polifenóis são substâncias químicas que possuem uma ou mais hidroxila ligadas ao seu anel aromático e são usados pelas plantas como forma de proteção (Lorenzi et al., 2002; Oliveira \& Mendes, 2016; Vera-Cruz et al., 2010).

A babosa é cultivada para fins medicinais e cosméticos, sendo que na medicina popular é mais usada por mulheres como forma de tratamento capilar, porém, é indicada como cicatrizante para queimaduras e ferimentos superficiais na pele. Em um experimento, quando testada em animais, foi capaz de acelerar a cicatrização e aumentar a proliferação celular (Freitas et al., 2014; Lorenzi et al., 2002). O capimsanto, também denominado como capim-cheiroso, vervena e capim limão tem sua utilização na prevenção e no tratamento de patologias, tais como, no controle de parasitas em rebanhos leiteiros, sendo uma maneira de manter não só as técnicas, mas as tecnologias de elaboração de substâncias necessárias para o manejo do rebanho (Guimarães et al., 2011). A sua utilização para o controle do carrapato é indicada por meio de macerados da planta (raízes, rizomas, colmos e folhas) que estarão em infusão e logo em seguida, introduzidos nos animais (Heimerdinger et al., 2006). Conhecida com catnip, a ervade-gato, possui seu efeito relacionado a um de seus óleos essenciais, chamado nepetalactona, no qual passa pelas vias respiratórias até chegar no sistema nervoso central, atuando diretamente. Segundo a médica veterinária Cecília Vargas, a planta não atrai somente gatos e sim todos os felinos, fazendo efeito até em leões. É utilizada na medicina veterinária para acalmar o temperamento de animais mais agressivos e tratar animais apáticos (Tucker \& Tucker, 1988). Também é utilizada para tratar doenças humanas e muito encontrada em brinquedos de gatos, sendo utilizada para tratamentos depressivos de longo período e insônia. Não é considerada uma droga, não trazendo a dependência dela para o animal (Bernardi et al., 2010).

Dos $66(53,2 \%)$ que fizeram o tratamento, houve um viés de duas pessoas, no qual foram descartados para a pesquisa, pois não citaram as partes das plantas utilizadas, sendo assim foi o utilizado para os dados 64 entrevistados. No qual puderam utilizar mais de uma alternativa para marcar quais partes das plantas foram usadas. Dos 64 entrevistados foi obtido 82 respostas da pergunta sobre quais partes da planta foram utilizadas, sendo $58,5 \%$ de folhas, $13,4 \%$ de frutos, $9,8 \%$ de bulbo, $4,9 \%$ de sementes e $3,6 \%$ de cascas (dos frutos e do caule) e $8,5 \%$ responderam que não sabiam (Gráfico 4). $\mathrm{O}$ uso maior de folhas se deve por ser uma das partes que mais contêm princípio ativo e além de ajudar na preservação da planta (Coan \& Matias, 2014). No entanto, segundo Pereira et al. (2015), as sementes também possuem uma grande quantidade de princípios ativos, assim como as folhas. A utilização da casca do caule é bem mais destrutiva por causar danos no desenvolvimento e longevidade da planta (Santos et al., 2012). Há indicações de que seja usado por preferência folhas, cascas, planta inteira, fruto, raiz e outras partes (Mattos et al., 2015), isso demonstra que na literatura, as partes mais usadas para preparação de medicamentos são folhas e casca. É muito importante saber sempre qual foi a parte utilizada da planta, pois em muitos casos, não é a planta toda que contêm as propriedades desejadas (Yarza, 1982) e o desconhecimento se deve, talvez, por não saber como foi o modo de preparo.

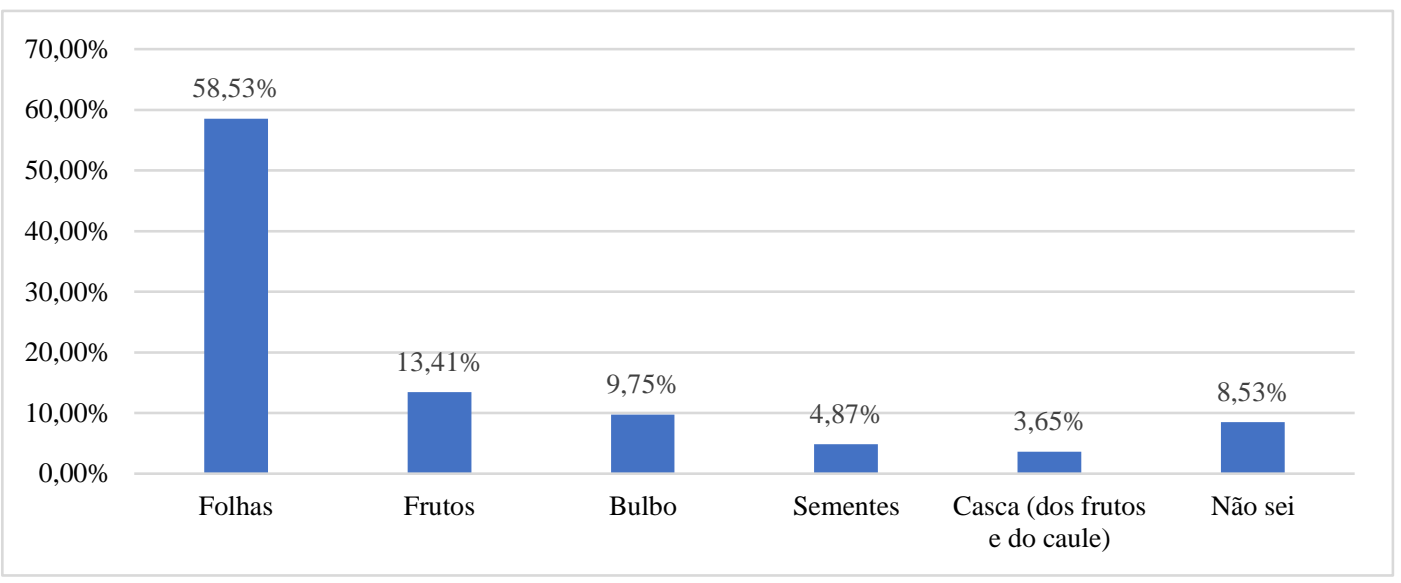

Gráfico 4: Partes das plantas que foram utilizadas para o tratamento (\%). 
Dentre os 66 entrevistados que utilizaram plantas medicinais como recurso terapêutico, houve um viés de três que não informaram quais doenças foram tratadas. Sendo assim, os dados utilizados foram dos 63 que informaram e citaram mais de duas formas de tratamento, dentre quais quatro doenças foram escolhidas por possuírem um maior índice de tratamento. No qual 23,2\% utilizaram para ansiedade; $12,8 \%$ utilizaram para problemas no fígado e estômago; $10,4 \%$ para inchaço e $6,4 \%$ para obesidade (Gráfico 5). Assim, como nos humanos, a ansiedade é muito comum nos animais de companhia e isso ocorre quando há ausência de figura de apego (Machado \& Sant'Anna, 2017). Como no caso de um cão da raça Bernese que se atirou do quarto andar do prédio em que vivia por estar sozinho no apartamento (Braitman, 2015). Algumas plantas atuam diretamente no sistema nervoso central, sendo utilizadas para o tratamento de transtornos como ansiedade e depressão, sendo uma delas a camomila e erva-de-gato (Almeida et al., 2013). Problemas no fígado e estômago nos animais ocorre principalmente por intoxicações, infecções virais ou bacterianas, além do uso exagerado de medicamentos. Algumas raças caninas possuem pré-disposição para esses problemas como Yorkshire e Maltês, assim como raças felinas: Himalaios e Persas. O uso do chá de boldo em animais ajuda a combater problemas no fígado (Cogliati et al., 2015). Inchaço pode ocorrer por conta de alguma alergia, seja a picada de inseto, vacinas, plantas e entre outros fatores e dependendo da localidade pode ser relacionado com um câncer. A arnica (Arnica montana) é uma das plantas indicadas como tratamento para esse tipo de caso, pois reduz o inchaço e traumatismo (Lorenzi et al., 2002). A obesidade é umas das doenças nutricionais mais comuns em cães, alterando funções corpóreas e limitando a longevidade do animal. Ela ocorre por diversos fatores como idade, pré-disposição de algumas raças, genética, falta de exercício e composições calóricas de alguns alimentos ingeridos pelo animal (Aptekmann et al., 2014). As plantas medicinais utilizadas para o tratamento de obesidade são em formas de medicamentos fitoterápicos (Manenti, 2010). A utilização de alcachofra (Cynara scolymus) reduz o peso do animal quando associado a atividade física (Correa et al., 2012).

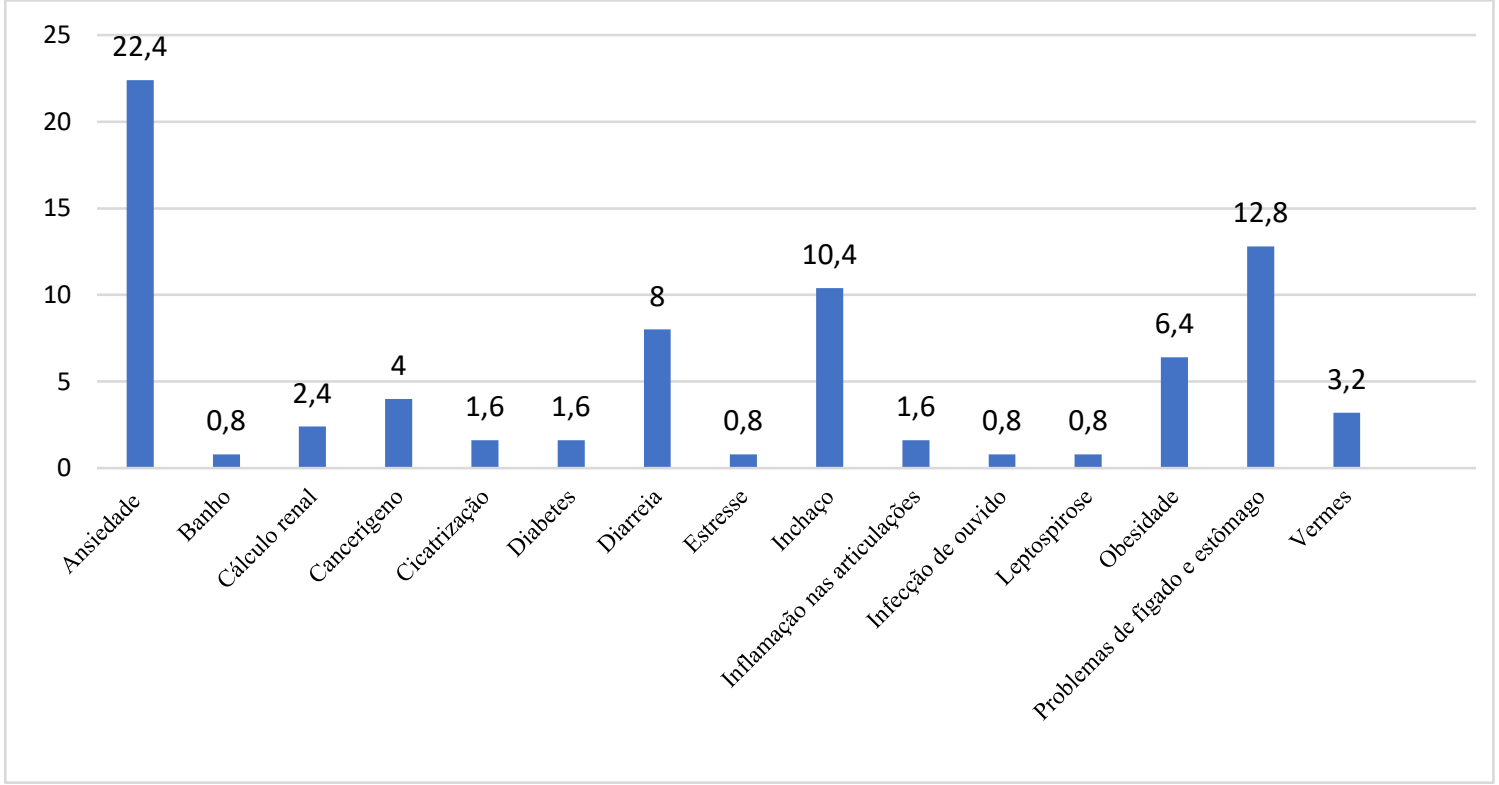

Gráfico 5. Índices de doenças tratadas por plantas medicinais e formas de tratamento (\%).

Dos entrevistados que fizeram tratamento, $18,8 \%$ continuam utilizando a planta, enquanto $16,6 \%$ utilizaram durante um mês e 15,1\% utilizaram durante um ano (Gráfico 6). Existem várias probabilidades que podem aumentar o longo uso do medicamento/planta como, por exemplo, a doença a ser tratada. Algumas plantas possuem um período para serem utilizadas, como a alcachofra (Cynara scolymus) que deve ser usada somente durante 30 dias e a Garcinia cambogia que deve ser utilizada somente durante 8 semanas, ambas são usadas para tratamentos para a obesidade (Correa et al., 2012). Além disso, não é recomendável o uso por um longo período de tempo, pois a planta pode perder a sua ação medicinal (Suzuki et al., 2020). Pela superdosagem e a utilização simultânea com outros medicamentos pode causar efeitos adversos (Taufner et al., 2006). 


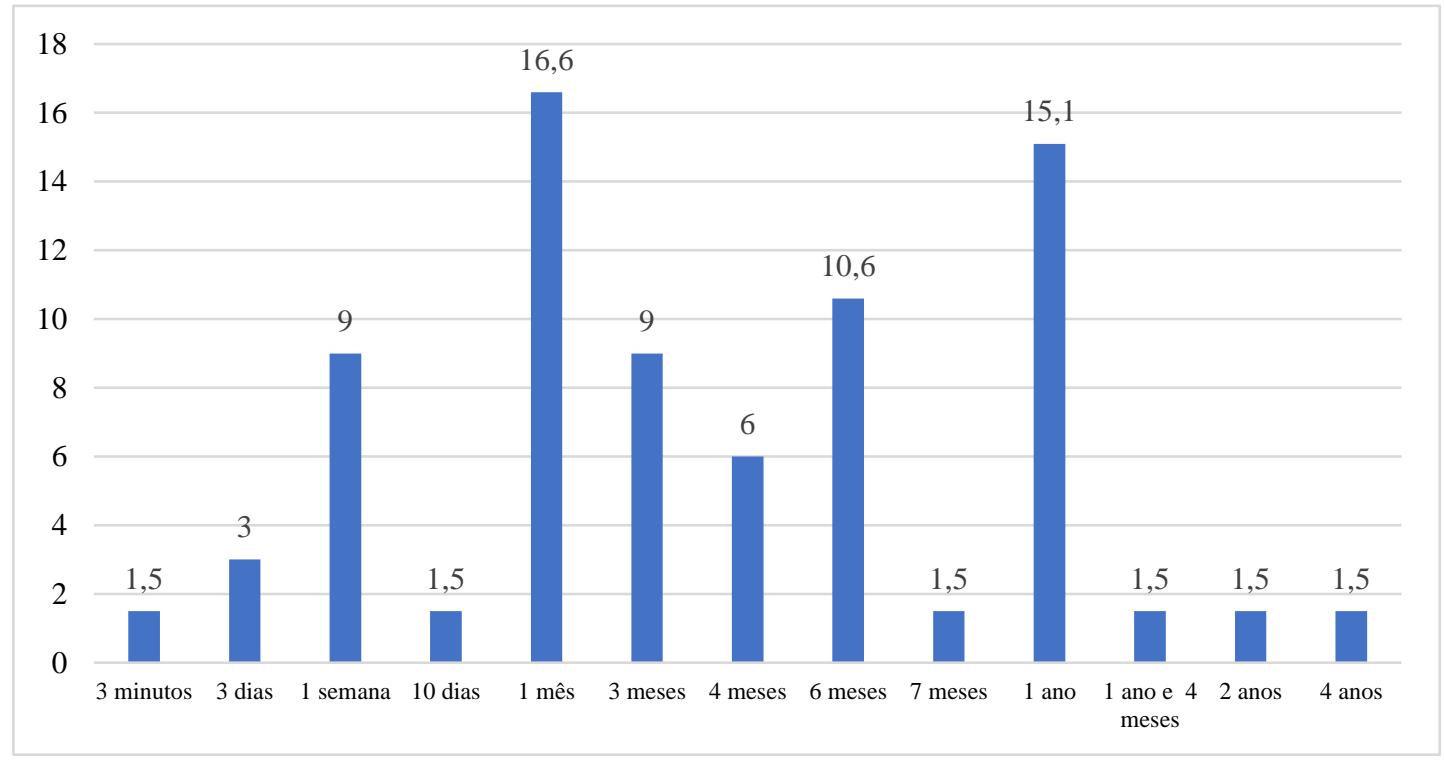

Gráfico 6. Período utilizado para os tratamentos (\%).

Todos os entrevistados que fizeram o tratamento receberam várias indicações, dentre elas, $54,5 \%$ tiveram indicações de famílias e amigos, enquanto 31,8\% tiveram por um médico veterinário, $9 \%$ por meio de grupos em redes sociais e $8,0 \%$ buscaram por artigos (Gráfico 7). De acordo com Ministério Único de Saúde (SUS), tratamentos a base de plantas medicinais e medicamentos fitoterápicos tiveram um alto índice de busca, pois, a população aprendeu a usá-los com seus avos e pais. Porém, é considerado automedicação quando não ocorre prescrição médica, sendo recomendável que seja evitado esse tipo de situação, mesmo que tenha sido buscado por livros ou artigos (Oliveira et al., 2014), já que a maioria dos profissionais de saúde, que sabem pouco sobre o assunto por não terem base em sua formação acadêmica, acabam não sabendo como lidar na pratica e mesmo que haja indicação por um veterinário deve-se tomar cuidado com as doses utilizadas (Lopes et al., 2012).

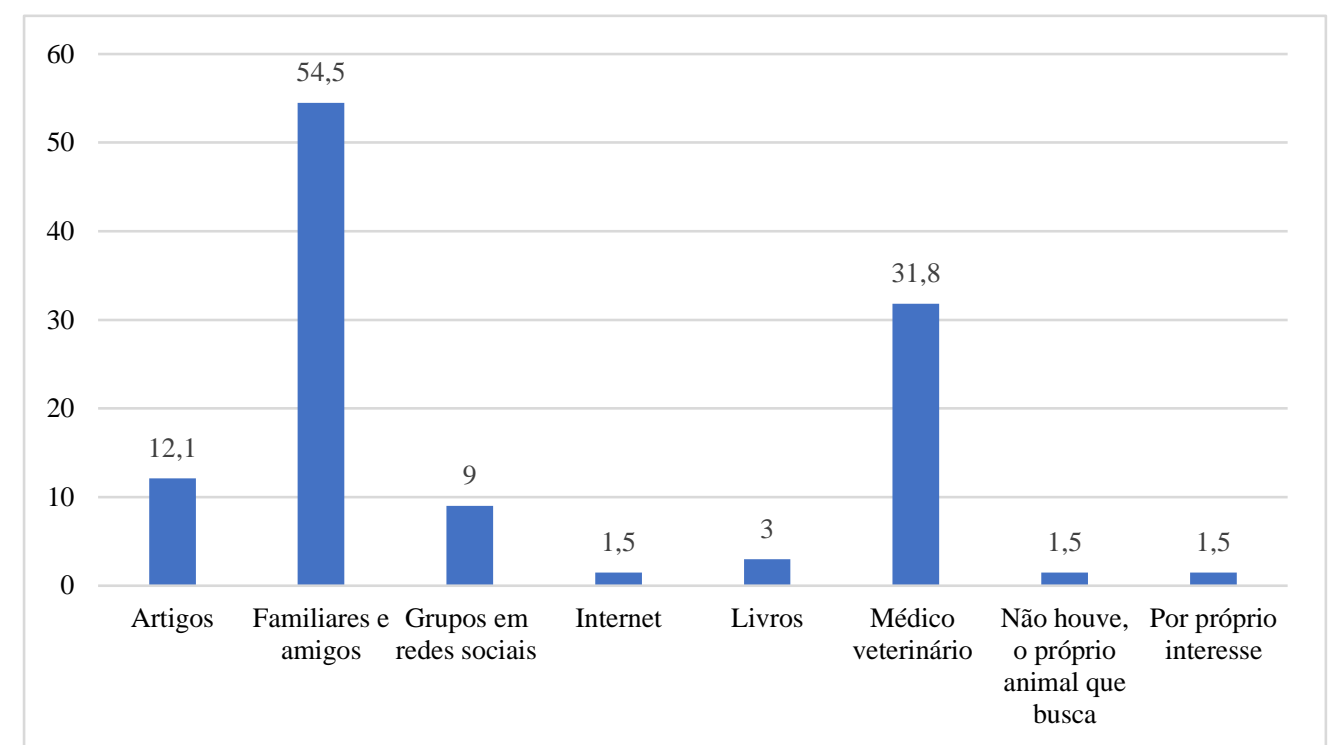

Gráfico 7: Porcentagem (\%) das indicações do tratamento à base de plantas medicinais por amigos, artigos, familiares e outros.

As plantas ou medicamentos foram adquiridos por meio de cultivo próprio $(36,0 \%)$, farmácia de manipulação $(28,8 \%)$, feiras $(18,0 \%)$, lojas $(8,0 \%)$ e plantado na quadra onde reside $(1,5 \%)$ (Gráfico 8). Antigamente, o ser humano só fazia coleta de plantas que nasciam espontaneamente em qualquer localidade. Após o crescimento absurdo de procura, o cultivo próprio de plantas começou a ser utilizado. O cultivo estabelece uma grande importância para a sustentabilidade do meio ambiente; porém, é recomendado a orientação sobre o cultivo e o manejo correto. Há um preparo essencial para a criação 
de uma horta própria, no qual primeiro deve-se ter conhecimento da espécie de planta na qual está trabalhando, manuseio durante e após a colheita (Brasileiro et al., 2008). O aumento pela procura de plantas em farmácia de manipulação é explicado pelo avanço de pesquisas cientificas comprovando o seu uso e criação de medicamentos fitoterápicos eficazes (Vieira et al., 2010). Há poucos estudos em relação às plantas medicinais sendo vendidas em feiras no Brasil; porém, se sabe que as plantas mais vendidas em feiras para obesidade são chá-verde, espinheira-santa (Maytenus ilicifolia) e cavalinha (Equisetum arvense) (Maioli-Azevedo \& Fonseca-Kruel, 2007; Morais et al., 2005).

Ao serem questionados se houve resultado no tratamento, um dos entrevistados não respondeu, havendo um viés. E dentre os 65 que responderam: 96,9\% disseram que sim e 3,1\% disseram que não (Figura 3). As respostas positivas se devem pelo fato que o uso de tratamentos à base de plantas medicinais tem gerado uma grande eficácia contra doenças (Santana et al., 2015). Enquanto as respostas negativas podem ter sido por vários fatores, como exemplo o uso incorreto da planta. Na maioria das vezes, ocorre a identificação errada de uma planta e isso pode piorar a saúde do animal (Silveira et al., $\underline{2013}$.

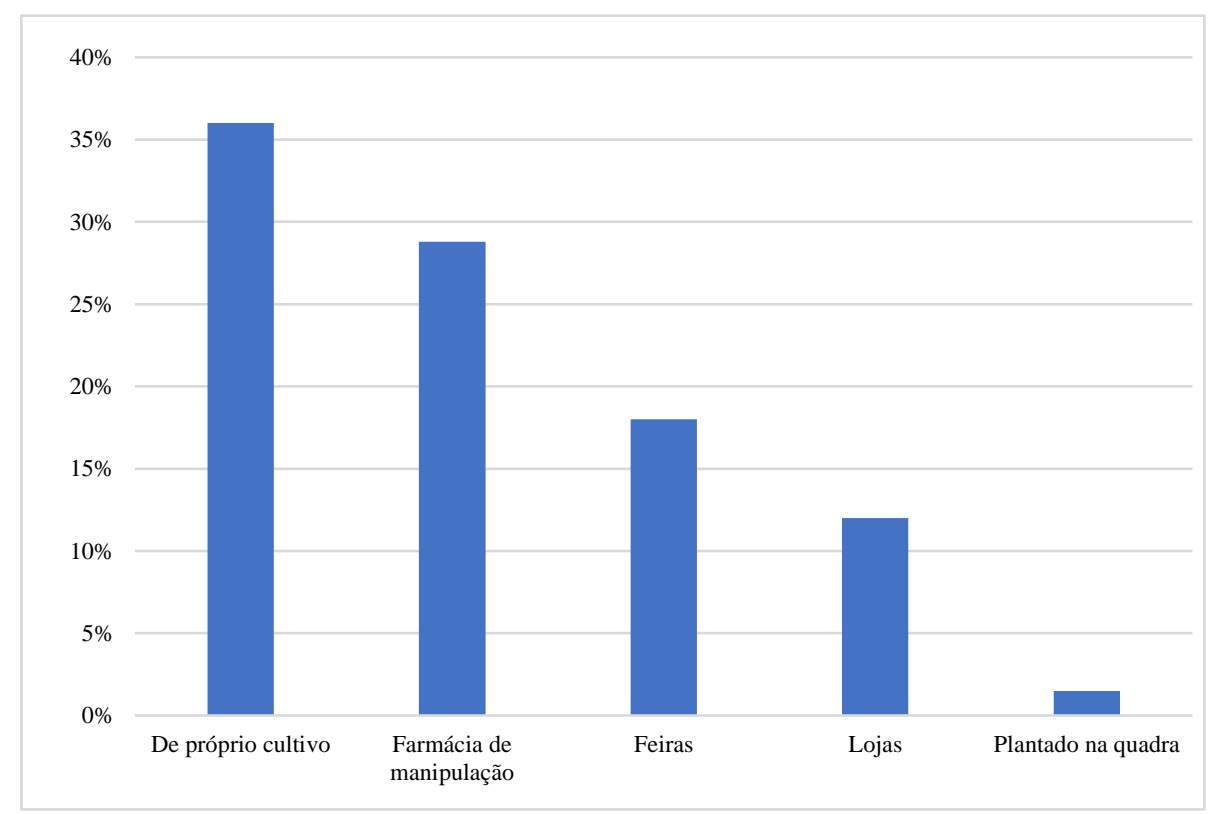

Gráfico 8. Locais em que as plantas foram adquiridas (\%).

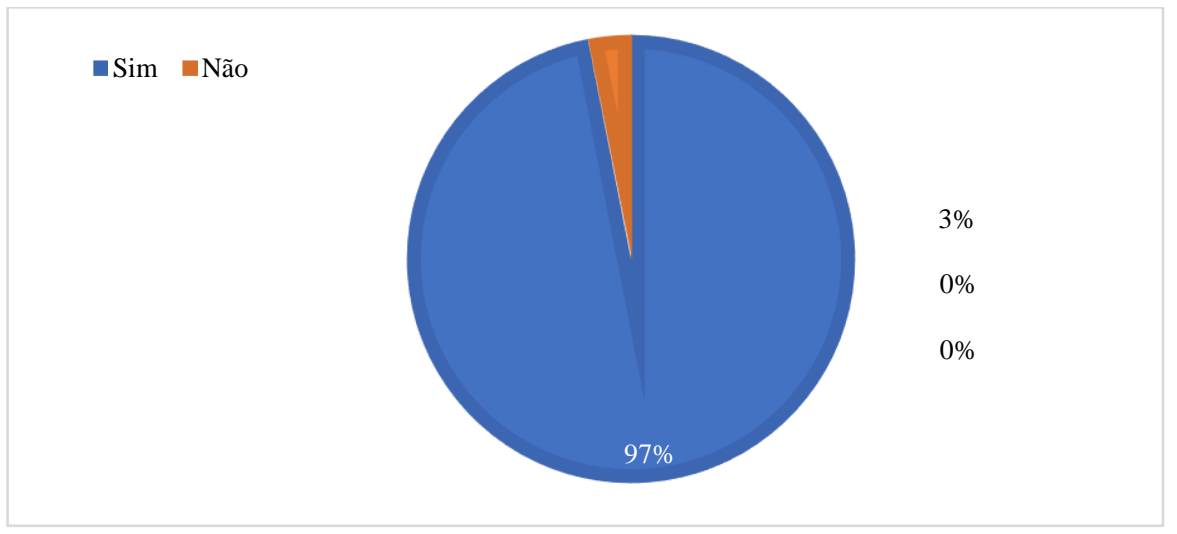

Figura 3. Resposta da pergunta sobre ter tido resultado ou não com o tratamento (\%).

Ao serem questionados por que escolheram esse tipo de tratamento e não medicamentos convencionais, $71,21 \%$ responderam que o tratamento era eficaz, $27,7 \%$ pela opção de preço, $9,0 \%$ leu na internet e 4,5\% por vídeos no YouTube (Gráfico 9).

Como já foi exposto em alguns momentos, o tratamento de plantas medicinais, tanto em humanos como animais, possui uma eficácia muito grande e já foi comprovado cientificamente. Segundo alguns médicos, os medicamentos à base de planta trazem um conforto emocional muito maior e podem ser 
uma opção para ocupar o lugar de medicamentos tradicionais (Balbino \& Dias, 2010). O uso de plantas medicinais como tratamento possuem um alto índice de uso por ser de baixo custo e de fácil acesso pela população (Zeni et al., 2017). É muito perigoso a utilização de plantas medicinais por conta própria baseada em pesquisas na internet, por isso em 2017, o Ministério Único da Saúde (SUS) disponibilizou em seu site AVASUS um curso gratuito sobre plantas medicinais e medicamentos fitoterápicos para agentes comunitários de saúde (Silvello, 2010).

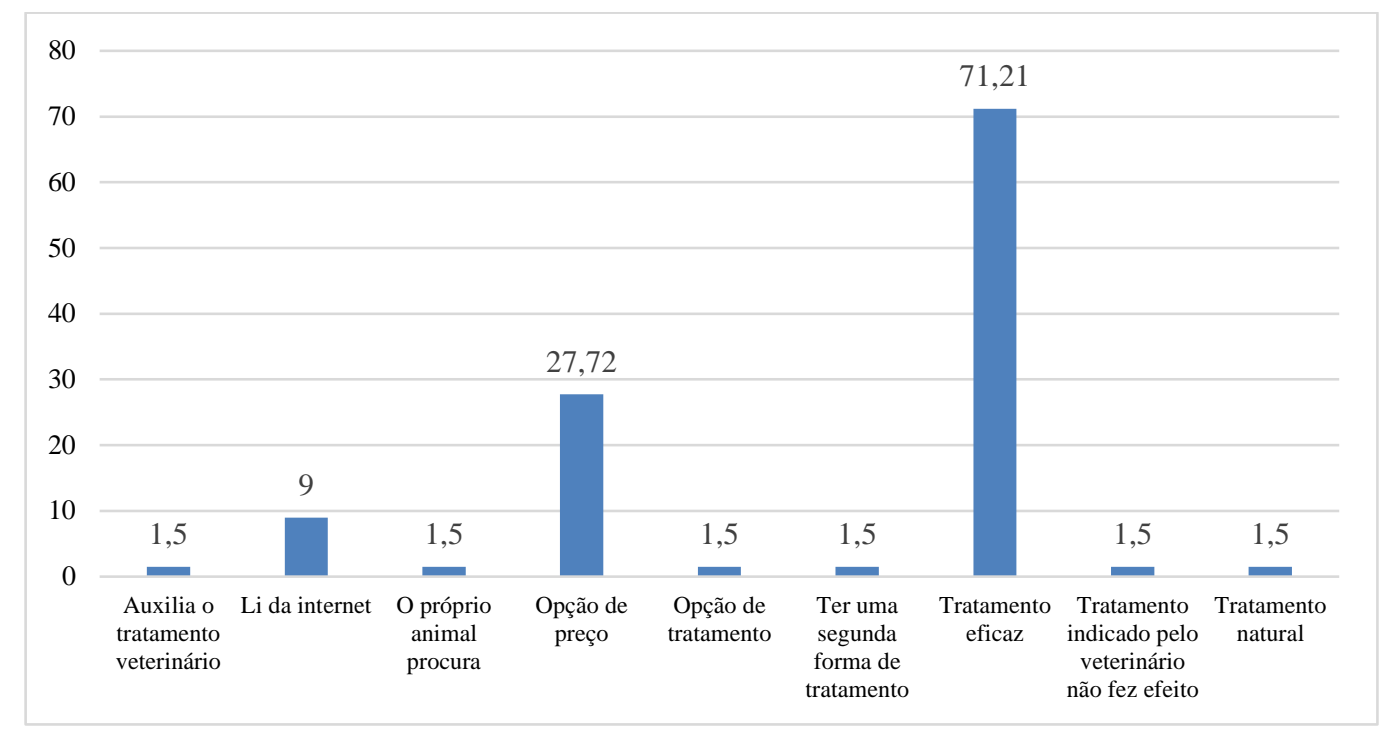

Gráfico 9: Porcentagem (\%) das escolhas pelo tratamento com plantas medicinais

\section{Conclusão}

Com este trabalho, pode-se observar a relevância das plantas medicinais na área da medicina veterinária e que este tratamento deve estar sempre associado há outro, pois, ele surgiu para complementar a medicina convencional já conhecida. E ressaltar que a falta de conhecimento da população e dos próprios servidores da área da saúde pode não só afetar a saúde pública, mas também o bem-estar animal e que isso pode ser melhorado com pesquisas científicas e a segregação de uma disciplina com este tema na grade curricular das instituições.

\section{Referências}

Almeida, A. A. C., de Carvalho, R. B. F., Coelho, M. L., \& de Freitas, R. M. (2013). Utilização de plantas medicinais para o tratamento da depressão: uma prospecção tecnológica. Revista GEINTECGestão, Inovação e Tecnologias, 3(2), 157-166. DOI: https://doi.org/10.7198/s2237$\underline{0722201300020013}$

Aptekmann, K. P., Suhett, W. G., Junior, A. F. M., Souza, G. B., Tristão, A. P. P. A., Adams, F. K., Aoki, C. G., Junior, R. J. G. P., Carciofi, A. C., \& Tinucci-Costa, M. (2014). Aspectos nutricionais e ambientais da obesidade canina. Ciência Rural, 44(11), 2039-2044. DOI: https://doi.org/10.1590/0103-8478cr20130524

Balbino, E. E., \& Dias, M. F. (2010). Farmacovigilância: um passo em direção ao uso racional de plantas medicinais e fitoterápicos. Revista Brasileira de Farmacognosia, 20(6), 992-1000. DOI: https://doi.org/10.1590/s0102-695x2010005000031

Batista, F. T., Lacerda, G. D., Silva, J. R. S., Rodrigues, L. F. T., Feitosa, T. P., \& Mustafa, V. S. (2017). O uso de plantas medicinais na medicina veterinária-riscos e benefícios. Revista Científica Do Curso de Medicina Veterinária, 4(2), 62-74.

Bernardi, M. M., Kirsten, T. B., Salzgeber, S. A., Ricci, E. L., Romoff, P., Lago, J. H. G., \& Lourenço, L. M. (2010). Antidepressant-like effects of an apolar extract and chow enriched with Nepeta cataria (catnip) in mice. Psychology \& Neuroscience, 3(2), 251. DOI: https://doi.org/10.3922/j.psns.2010.2.015 
Braitman, L. (2015). Animal Madness: Inside Their Minds. Nova Iorque: Simon \& Schuster. ISBN-10: 1451627017.

Brasileiro, B. G., Pizziolo, V. R., Matos, D. S., Germano, A. M., \& Jamal, C. M. (2008). Plantas medicinais utilizadas pela população atendida no" Programa de Saúde da Família", Governador Valadares, MG, Brasil. Revista Brasileira de Ciências Farmacêuticas, 44(4), 629-636. DOI: https://doi.org/10.1590/s1516-93322008000400009

Carvalho, A. C. B., Balbino, E. E., Maciel, A., \& Perfeito, J. P. S. (2008). Situação do registro de medicamentos fitoterápicos no Brasil. Revista Brasileira de Farmacognosia, 18(2), 314-319. DOI: https://doi.org/10.1590/s0102-695x2008000200028

Coan, C. M., \& Matias, T. (2014). A utilização das plantas medicinais pela comunidade indígena de Ventarra Alta-RS. SaBios-Revista de Saúde e Biologia, 9(1), 11-19.

Cogliati, B., Silva, R. D., \& Ushikoshi, W. S. (2015). Doenças hepáticas caninas. In M. M. Jerico, J. P. Andrade Neto, \& M. M. Kogika (Eds.), Tratado de medicina interna de cães e gatos. Rio de Janeiro: Roca, vol.1 v. p. 1035-1043. (Vol. 1, pp. 1035-1043). Roca.

Correa, E. C. M., Santos, J. M., \& Ribeiro, P. L. B. (2012). Uso de fitoterápicos no tratamento da obesidade uma revisão de literatura. In Monografia. Pontifícia Universidade Católica de Goiás.

Firmo, W. da C. A., Menezes, V. J. M., Passos, C. E. C., Dias, C. N., Alves, L. P. L., Dias, I. C. L., Neto, M. S., \& Olea, R. S. G. (2012). Contexto histórico, uso popular e concepção científica sobre plantas medicinais. Cadernos de Pesquisa, 18, 90-95.

Freitas, V. S., Rodrigues, R. A. F., \& Gaspi, F. O. G. (2014). Propriedades farmacológicas da Aloe vera (L.) Burm. f. Revista Brasileira de Plantas Medicinais, 16(2), 299-307.

Gonçalves, G. A., Sá-Nakanishi, A. B., Wendt, M. M. N., Comar, J. F., Amado, C. A. B., Bracht, A., \& Peralta, R. M. (2015). Green tea extract improves the oxidative state of the liver and brain in rats with adjuvant-induced arthritis. Food \& Function, 6(8), 2701-2711. DOI: https://doi.org/10.1039/c5fo00548e

Guimarães, L. G. L., Cardoso, M. G., Sousa, P. E., Andrade, J., \& Vieira, S. S. (2011). Atividades antioxidante e fungitóxica do óleo essencial de capim-limão e do citral. Revista Ciência Agronômica, 42(2), 464-472. DOI: https://doi.org/10.1590/s1806-66902011000200028

Haraguchi, L. M. M., \& Carvalho, O. B. (2010). Plantas medicinais. In Plantas medicinais do curso de plantas medicinais.

Heimerdinger, A., Olivo, C. J., Molento, M. B., Agnolin, C. A., Ziech, M. F., Scaravelli, L. F. B., Skonieski, F. R., Both, J. F., \& Charão, P. S. (2006). Extrato alcoólico de Capim-cidreira (Cymbopogon citratus) no controle do Boophilus microplus em bovinos. Revista Brasileira de Parasitologia Veterinária, 15(1), 37-39.

Lameira, O. A. (2008). Plantas medicinais: do cultivo, manipulação e uso à recomendação popular. Embrapa Amazônia Oriental.

Lopes, M. A., Obici, S., \& Albiero, A. L. M. (2012). Conhecimento e intenção de uso da fitoterapia em uma Unidade Básica de Saúde. Interfaces Científicas-Saúde e Ambiente, 1(1), 53-59. DOI: https://doi.org/10.17564/2316-3798.2012v1n1p53-59

Lorenzi, H., Matos, F. J., \& Francisco, J. M. (2002). Plantas medicinais no Brasil: nativas e exóticas (Vol. 1).

Lourenzani, A., Lourenzani, W. L., \& Batalha, M. O. (2004). Barreiras e oportunidades na comercialização de plantas medicinais provenientes da agricultura familiar. Informações Econômicas, 34(3), 15-25.

Machado, D. S., \& Sant'Anna, A. C. (2017). Síndrome de ansiedade por separação em animais de companhia: Uma revisão. Revista Brasileira de Zoociências, 18(3). DOI: https://doi.org/10.34019/2596-3325.2017.v18.24682

Maioli-Azevedo, V., \& Fonseca-Kruel, V. S. (2007). Plantas medicinais e ritualísticas vendidas em feiras livres no Município do Rio de Janeiro, RJ, Brasil: estudo de caso nas zonas Norte e Sul. Acta Botanica Brasilica, 21(2), 263-275. DOI: https://doi.org/10.1590/s0102-33062007000200002

Manenti, A. V. (2010). Plantas medicinais utilizadas no tratamento da obesidade: uma revisão. 
Universidade do Extremo Sul Catarinense.

Mattos, A. A., Morais, R. B., Sampaio, J. B., Lacerda, G. A., Ramos, D. M. B., \& Carreiro, D. L. (2015). Levantamento de plantas medicinais comercializadas por raizeiros do mercado municipal central de Montes Claros-MG. Revista Brasileira de Pesquisa Em Ciências Da Saúde, 2(1), 11-17.

Monteiro, M. V. B., Rodrigues, S. T., \& Vasconcelos, A. L. F. (2012). Plantas medicinais utilizadas na medicina etnoveterinária praticada na ilha do Marajó. Embrapa Amazônia Oriental, 380, 1-33.

Morais, S. M., Dantas, J. D. P., Silva, A. R. A., \& Magalhães, E. F. (2005). Plantas medicinais usadas pelos índios Tapebas do Ceará. Brazilian Journal of Pharmacognosy, 15(2), 169-177. DOI: https://doi.org/10.1590/s0102-695x2005000200017

Oliveira, L. A. R., Machado, R. D., \& Rodrigues, A. J. L. (2014). Levantamento sobre o uso de plantas medicinais com a terapêutica anticâncer por pacientes da Unidade Oncológica de Anápolis. Revista Brasileira de Plantas Medicinais, 16(1), 32-40. DOI: https://doi.org/10.1590/s1516$\underline{05722014000100005}$

Oliveira, N. C., \& Mendes, D. R. G. (2016). As Propriedades da Camellia sinensis (Chá verde). Plants Rescue.

Ozaki, A. T., \& Duarte, P. C. (2006). Fitoterápicos utilizados na medicina veterinária, em cães e gatos. Revista Pharmacia Brasileira. Infarma, 18(11-12), 17-25.

Pateiro, M., Lorenzo, J. M., Amado, I. R., \& Franco, D. (2014). Effect of addition of green tea, chestnut and grape extract on the shelf-life of pig liver pâté. Food Chemistry, 147(0), 386-394. DOI: https://doi.org/10.1016/j.foodchem.2013.09.153

Pereira, J. B. A., Rodrigues, M. M., Morais, I. R., Vieira, C. R. S., Sampaio, J. P. M., Moura, M. G., Damasceno, M. F. M., Silva, J. N., Calou, I. B. F., \& Deus, F. A. (2015). O papel terapêutico do Programa Farmácia Viva e das plantas medicinais. Revista Brasileira de Plantas Medicinais, 17(4), 550-561. DOI: https://doi.org/10.1590/1983-084x/14_008

Santana, D. C., Souza, T. S., Pierro, P. C. C., Amaral, A. A., \& Alegre, A. (2015). Uso de plantas medicinais na criação animal. Enciclopédia Biosfera, 11, 96-98.

Santos, S. L. D. X., Alves, R. R. N., Barbosa, J. A. A., \& Brasileiro, T. F. (2012). Plantas utilizadas como medicinais em uma comunidade rural do semi-árido da Paraíba, Nordeste do Brasil. Revista Brasileira de Farmácia, 93(1), 68-79.

Scholl, A. L., \& Ricardo, K. R. (2010). Conhecimento popular sobre o uso de plantas medicinais. Caxias Do Sul.

Silva, F. de J., Silveira, A. P., \& Gomes, V. S. (2016). Plantas medicinais e suas indicações ginecológicas: estudo de caso com moradoras de Quixadá, CE, Brasil. Revista Brasileira de Biociências, 14(3), 193-201.

Silva, V. S., Ribeiro, J. R. S., Alves, T. N., Silva, P. H. S., \& Rosal, L. F. (2013). Uso e diversidade de plantas medicinais na Comunidade de Curuperé, Município de Curuçá-PA. Cadernos de Agroecologia, 8(2).

Silveira, M. A., Lassen, M. F. M., \& Beuter, S. B. (2013). Utilização das plantas medicinais e fitoterápicas o conhecimento popular em uma revisão bibliográfica histórica. XXI Seminário de Iniciação Científica.

Silvello, C. L. C. (2010). O uso de plantas medicinais e de fitoterápicos no SUS: uma revisão bibliográfica. Universidade Federal do Rio Grande do Sul.

Simoni, I. C. (2011). Plantas com poder curativo na saúde animal. Instituto Biológico, 155.

Sousa, V. F. O., Bandeira, A. S., Ribeiro, M. D. S., Santos, J. J. F., Santos, G. L., Silva, R. A., Maracajá, P. B., \& Costa, J. E. (2020). Uso de fitoterápicos na cura de enfermidades em animais no Semiárido Paraibano. Research, Society and Development, 9(7), e261974040-e261974040. DOI: https://doi.org/10.33448/rsd-v9i7.4040

Suzuki, A. L. M., Baroni, C., Ranucci, J. S. T., \& Martins, L. A. (2020). Cartilha de plantas medicinais: indicadas para alívio de sintomas respiratórios. PROREXT/UFRGS.

Taufner, C. F., Ferraço, E. B., \& Ribeiro, L. F. (2006). Uso de plantas medicinais como alternativa 
fitoterápica nas unidades de saúde pública de Santa Teresa e Marilândia, ES3. Natureza, 4(1), 3039.

Teixeira, V. H. S., Fernandes, N. C. F., Corrêa, F. C., \& Oliveira, D. M. C. (2015). Plantas medicinas na etnoveterinária no Brasil - Artigo de revisão. Anclivepa, 42, 926-930.

Tucker, A. O., \& Tucker, S. S. (1988). Catnip and the catnip response. Economic Botany, 42(2), 214231. DOI: https://doi.org/10.1007/bf02858923

Vera-Cruz, M., Nunes, E., Mendonça, L., Chaves, É., \& Fernandes, M. L. L. A. (2010). Efeito do chá verde (Camelia sinensis) em ratos com obesidade induzida por dieta hipercalórica. Jornal Brasileiro de Patologia e Medicina Laboratorial, 46(5), 407-413. DOI: https://doi.org/10.1590/s1676$\underline{24442010000500010}$

Vieira, J. N., Coelho, E. G. A., \& Oliveira, D. A. A. (2009). Sexagem molecular em aves silvestres. Revista Brasileira de Reprodução Animal, 33(2), 66-70.

Vieira, S. C. H., Sólon, S., Vieira, M. do C., \& Zárate, N. A. H. (2010). Levantamento de fitoterápicos manipulados em farmácias magistrais de Dourados-MS. Revista Brasileira de Farmacognosia, 20(1), 28-34. DOI: https://doi.org/10.1590/s0102-695x2010000100007

Yarza, O. (1982). Plantas que curam and plantas que matam: anatomia, enfermidades, processos curativos vegetais, plantas narcóticas, um cientista das plantas e das ervas. Ryoki Inoue Produções.

Zeni, A. L. B., Parisotto, A. V., Mattos, G., \& Helena, E. T. S. (2017). Utilização de plantas medicinais como remédio caseiro na Atenção Primária em Blumenau, Santa Catarina, Brasil. Ciência \& Saúde Coletiva, 22(8), 2703-2712. DOI: https://doi.org/10.1590/1413-81232017228.18892015

Histórico do artigo:

Recebido: 14 de outubro de 2020.

Aprovado: 24 de novembro de 2020.

Disponível online: 2 de março de 2021
Licenciamento: Este artigo é publicado na modalidade Acesso Aberto sob a licença Creative Commons Atribuição 4.0 (CC-BY 4.0), a qual permite uso irrestrito, distribuição, reprodução em qualquer meio, desde que o autor e a fonte sejam devidamente creditados. 